\title{
Totipotent Neuroepithelial Stem Cell
}

National Cancer Institute

\section{Source}

National Cancer Institute. Totipotent Neuroepithelial Stem Cell. NCI Thesaurus. Code C33796.

A relatively undifferentiated cell that retains the ability to divide into any type of nerve cell. 